\title{
Quantum information processing with trapped ions
}

\author{
By D. J. Wineland, M. Barrett, J. Britton, J. Chiaverini, \\ B. Demarco, W. M. Itano, B. Jelenković ${ }^{\dagger}$, C. Langer, D. Leibfried, \\ V. Meyer, T. Rosenband, and T. Schätz \\ Time and Frequency Division \\ National Institute of Standards and Technology \\ Boulder, CO, 80305-3328, USA \\ $\dagger$ permanent address: Institute of Physics, Belgrade, Yugoslavia
}

Experiments directed towards the development of a quantum computer based on trapped atomic ions are described briefly. We discuss the implementation of single qubit operations and gates between qubits. A geometric phase gate between two ion qubits is described. Limitations of the trapped-ion method such as those caused by Stark shifts and spontaneous emission are addressed. Finally, we describe a strategy to realize a large-scale device.

Keywords: quantum information processing, quantum computation, entanglement, trapped atomic ions

\section{Introduction}

Efforts to realize experimentally the elements of quantum computation (QC) using trapped atomic ions have been stimulated in large part by a proposal by Cirac and Zoller (1995). In this scheme, ions confined in a linear RF (Paul) trap are cooled and form a stable spatial array whose motion is described by normal modes. Two internal levels in each ion form a qubit (referred to below as a spin qubit). The spacing between ions is typically large enough $(>1 \mu \mathrm{m})$ that the direct coupling of internal states of ions is negligible, thereby precluding logic gates based on internalstate interactions. (An exception to this might be "Dipole blockade" gates based on Rydberg transitions as envisioned for neutral atoms (Jaksch et al. 2000), but these gates are experimentally more challenging for ions because of the higher energies between ground and Rydberg levels.) To overcome this limitation, Cirac and Zoller suggested cooling the ions to their motional ground state and using the ground and first excited state of a particular collective motional mode as a qubit (motion qubit). The motional mode can act as a data bus to transfer information between ions by mapping the spin qubit state of a particular ion onto the selected motion qubit with a laser beam focused onto that ion. The ability to construct a logic gate between the motion qubit and another selected spin qubit, coupled with the ability to perform single-spin qubit rotations provides the basis for universal quantum computation (DiVincenzo 1995, Barenco et al. 1995).

The ion-trap scheme satisfies the main requirements for a quantum computer as outlined by DiVincenzo (2001): (1) a scalable system of well-defined qubits, 
(2) a method to reliably initialize the quantum system, (3) long coherence times,

(4) existence of universal gates, and (5) an efficient measurement scheme. Most of these requirements have been demonstrated experimentally; consequently, ion trap quantum processors are studied in several laboratories. Here, we focus on experiments carried out at NIST but note that similar work is being pursued at Aarhus, Almaden (IBM), Hamburg, Hamilton (McMaster Univ., Ontario), Innsbruck, Los Alamos (LANL), University of Michigan, Garching (MPI), Oxford, and Teddington (NPL, U.K.).

\section{Coherent control and entanglement}

The key entangling operation in the 1995 Cirac/Zoller scheme for QC, and other schemes that rely on the ions' motion, is an operation that couples a spin qubit with the motion in a spin-state-dependent way. Assume that the spin qubit has a ground state labeled $|\downarrow\rangle$ and a higher metastable state labeled $|\uparrow\rangle$ that are separated in energy by $\hbar \omega_{0}$. First assume that single-photon transitions between these levels can be excited with a focused laser beam. For simplicity, we will assume electric dipole transitions, but this is easily adapted to other cases such as electric quadrupole transitions as in the Innsbruck experiments (Roos et al. 1999). The interaction between an ion and the electric field of the laser beam can be written as

$$
H_{I}(t)=-\vec{d} \cdot \vec{E}=-\vec{d} \cdot E_{0} \hat{\epsilon}_{L} \cos \left(k \widetilde{z}-\omega_{L} t+\phi\right),
$$

where $\vec{d}$ is the electric dipole operator, $\widetilde{z}$ is the ion position operator for displacements from the ion's equilibrium position (expanded in terms of normal mode operators), $\hat{\epsilon}_{L}$ is the laser beam polarization, $k$ is the laser beam's $k$-vector (taken to be parallel to $\hat{z}$, the axis of the trap), $\omega_{L}$ is the laser frequency, and $\phi$ is the phase of the laser field at the mean position of the ion. $E_{0}$ is the laser beam electric field amplitude at the ion, which is assumed to be classical. We characterize the laser field's polarization with respect to a magnetic field $\vec{B}_{0}$ that sets the quantization axis for the ions. The polarization is expressed in terms of left circular $\left(\hat{\sigma}_{-}\right)$, linear $(\hat{\pi})$, and right circular $\left(\hat{\sigma}_{+}\right)$polarizations so that $\hat{\epsilon}_{L}=e_{-} \hat{\sigma}_{-}+e_{0} \hat{\pi}+e_{+} \hat{\sigma}_{+}$ with $\left|e_{-}\right|^{2}+\left|e_{0}\right|^{2}+\left|e_{+}\right|^{2}=1$. The dipole operator $\vec{d}$ is proportional to $\sigma^{+}+\sigma^{-}$, where $\sigma^{+} \equiv|\uparrow\rangle\left\langle\downarrow\left|, \sigma^{-} \equiv\right| \downarrow\right\rangle\langle\uparrow|$, and we take $\widetilde{z}=z_{0}\left(a+a^{\dagger}\right)$, where $a$ and $a^{\dagger}$ are the lowering and raising operators for the harmonic oscillator associated with the selected motional mode (frequency $\omega_{z}$ ) and $z_{0}$ is the extension of the ground-state wavefunction for the particular ion (and mode) being addressed. We assume all other $z$ modes are cooled to and remain in their ground states and for simplicity have neglected them in $\widetilde{z}$. In the Lamb-Dicke limit, where the extent of the ion's motion is much less than $\lambda / 2 \pi=1 / k$, we can write Eq. (2.1) (in the interaction frame, and making the rotating wave approximation (see for example, Wineland $e t$ al. 1998)) as

$$
H_{I} \simeq \hbar\left(\Omega e^{i \phi}\right) \sigma^{+} e^{-i\left(\omega_{L}-\omega_{0}\right) t}\left[1+i \eta\left(a e^{-i \omega_{z} t}+a^{\dagger} e^{i \omega_{z} t}\right)\right]+\text { h.c. }
$$

Here, $\Omega \equiv-E_{0}\left\langle\uparrow\left|\vec{d} \cdot \hat{\epsilon}_{L}\right| \downarrow\right\rangle /(2 \hbar)$ and $\eta \equiv k z_{0}$ is the Lamb-Dicke parameter ( $\ll 1$ in the Lamb-Dicke limit).

For certain choices of $\omega_{L}, H_{I}$ is resonant and the spin and motion can be coupled efficiently. For example, when $\omega_{L}=\omega_{0}-\omega_{z}, H_{I} \simeq i \eta \hbar\left(\Omega e^{i \phi}\right) \sigma^{+} a+$ h.c.. 
This is usually called the "red-sideband" coupling and is formally equivalent to the Jaynes-Cummings Hamiltonian from quantum optics (see, for example, Raimond et al. 2001). Here, $|\downarrow\rangle \rightarrow|\uparrow\rangle$ transitions are accompanied by $|n\rangle \rightarrow|n-1\rangle$ motional mode transitions. When $\omega_{L}=\omega_{0}+\omega_{z}$ (the blue sideband frequency), $H_{I} \simeq i \eta \hbar\left(\Omega e^{i \phi}\right) \sigma^{+} a^{\dagger}+$ h.c., and $|\downarrow\rangle \rightarrow|\uparrow\rangle$ transitions are accompanied by $|n\rangle \rightarrow|n+1\rangle$ transitions. When $\omega_{L}=\omega_{0}, H_{I} \simeq \hbar\left(\Omega e^{i \phi}\right) \sigma^{+}+h . c$. and $|\downarrow\rangle \rightarrow|\uparrow\rangle$ transitions do not change $n$. These "carrier" transitions are used to perform the single spin qubit rotations.

\section{(a) Raman transitions}

Some experiments use two ground-state hyperfine levels as a qubit. Coherent transitions between these levels can be implemented with two laser beams that drive two-photon stimulated-Raman transitions. In this case, in Eq. (2.2), $k$ must

be replaced by the difference $\Delta k=\left|\vec{k}_{1}-\vec{k}_{2}\right|$ between $k$-vectors for the two Raman beams (again assumed to parallel to $\hat{z}$ ), $\omega_{L}$ and $\phi$ are replaced by $\omega_{1}-\omega_{2}$ and $\phi_{1}-\phi_{2}$, the frequency difference and phase difference between the laser beams at the mean position of the ion, and $\Omega e^{i \phi}$ is replaced by the resonant two-photon Raman Rabi rate

$$
\Omega e^{i \phi} \leftrightarrow \frac{e^{i\left(\phi_{1}-\phi_{2}\right)}}{4 \hbar^{2}} \sum_{i}\left\langle\uparrow\left|\vec{d} \cdot E_{2} \hat{\epsilon}_{2}\right| i\right\rangle\left\langle i\left|\vec{d} \cdot E_{1} \hat{\epsilon}_{1}\right| \downarrow\right\rangle / \Delta_{i} .
$$

In this expression, the subscripts denote the two laser beams, $|i\rangle$ are the (virtual, electronically excited) intermediate states of the Raman process, and $\Delta_{i}$ are the detunings of the Raman beams as indicated in Fig. 1. We have assumed $\Delta_{i} \gg \gamma_{i}$ where $\gamma_{i}$ are the decay rates from the intermediate states. With these substitutions, Eq. (2.2) applies in the Lamb-Dicke limit where the Lamb-Dicke parameter is now given by $\eta \equiv \Delta k z_{0} \ll 1$. For brevity, we will specialize to the stimulated-Ramantransition case in what follows.

When the difference frequency of the Raman beams is tuned to resonance with the red or blue sidebands or carrier, the interaction leads to the coherent evolution

$$
|\downarrow\rangle|n\rangle \rightarrow \cos \left(\Omega_{n, n^{\prime}} t\right)|\downarrow\rangle|n\rangle-i e^{i \phi} \sin \left(\Omega_{n, n^{\prime}} t\right)|\uparrow\rangle\left|n^{\prime}\right\rangle
$$

and

$$
|\uparrow\rangle|n\rangle \rightarrow-i e^{-i \phi} \sin \left(\Omega_{n, n^{\prime}} t\right)|\downarrow\rangle|n\rangle+\cos \left(\Omega_{n, n^{\prime}} t\right)|\uparrow\rangle\left|n^{\prime}\right\rangle .
$$

When $n^{\prime}=n \pm 1$ (blue or red sidebands), $\Omega_{n, n^{\prime}} \equiv \eta \Omega\left(n_{>}\right)^{1 / 2}$, where $n_{>}$is the greater of $n$ or $n^{\prime}$. In Fig. 1, we show a red sideband transition for $n=1$. When the duration of this operation is adjusted to give a $\pi$ pulse, the spin qubit to motion qubit mapping step required in the Cirac/Zoller scheme $(\alpha|\downarrow\rangle+\beta|\uparrow\rangle)|0\rangle \rightarrow|\downarrow\rangle(\alpha|0\rangle+\beta|1\rangle)$ is executed. From expressions (2.4) and (2.5) the entanglement between the spin and motion is evident since the final state cannot in general be factored into the product of spin and motional wavefunctions. Carrier transitions can also be described by these expressions where $n=n^{\prime}$ and $\Omega_{n, n^{\prime}}=\Omega$. For each ion we are free to choose $\phi=\phi_{1}-\phi_{2}=0$ but the phase of all operations on this ion must be referenced to this choice.

In order to keep the duration of an entangling operation relatively short, $\Omega_{n, n^{\prime}}$ for $n \neq n^{\prime}$ cannot be too small relative to $\Omega_{n, n}$. Therefore $\eta$ must be chosen large 


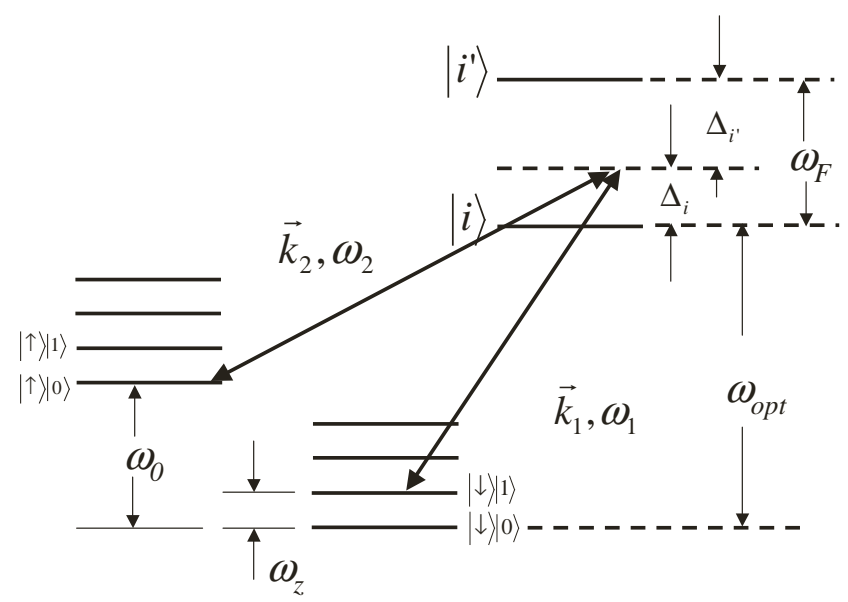

Figure 1. Schematic representation of relevant energy levels for stimulated-Raman transitions (not to scale). Shown are two ground-state hyperfine levels $(|\downarrow\rangle$ and $|\uparrow\rangle)$ for one ion, two (of possibly many) excited levels $\left(|i\rangle\right.$ and $\left|i^{\prime}\right\rangle$ ), and the harmonic oscillator levels for one mode of motion. Typically, $\omega_{z} \ll \omega_{0} \ll \Delta_{i}, \Delta_{i^{\prime}}, \omega_{F} \ll \omega_{\text {opt }}$ where $\omega_{\text {opt }}$ is an optical frequency.

enough that our interaction does not rigorously satisfy the Lamb-Dicke criterion and correction factors must be added to the expressions for the Rabi frequencies $\Omega_{n, n^{\prime}}$. These correction factors, which can be called Debye-Waller factors (Wineland et al. 1998), have been observed by Meekhof et al. (1996) and also form the basis for a controlled-not (CNOT) gate between motion and spin (Monroe et al. 1997, DeMarco et al. 2002).

\section{(b) Rabi frequencies}

For convenience of notation, in the case $\omega_{1}-\omega_{2} \simeq \omega_{0}$, we will designate $\omega_{1}=$ $\omega_{b}$ the "blue" Raman beam frequency and $\omega_{2}=\omega_{r}$ as the "red" Raman beam frequency. Similarly, we let $E_{1} \hat{\epsilon}_{1}=E_{b} \hat{\epsilon}_{b}=E_{b}\left(b_{-} \hat{\sigma}_{-}+b_{0} \hat{\pi}+b_{+} \hat{\sigma}_{+}\right)$and $E_{2} \hat{\epsilon}_{2}=$ $E_{r} \hat{\epsilon_{r}}=E_{r}\left(r_{-} \hat{\sigma}_{-}+r_{0} \hat{\pi}+r_{+} \hat{\sigma}_{+}\right)$. As an example relevant to the NIST experiments, we consider Raman transitions between the $2 s^{2} S_{1 / 2}$ hyperfine states $\mid F=2, m_{F}=$ $2\rangle \equiv|\downarrow\rangle$ and $\left|F=1, m_{F}=1\right\rangle \equiv|\uparrow\rangle$ in ${ }^{9} \mathrm{Be}^{+}$where we couple through the ion's $2 p$ fine structure levels. (In this example, $|i\rangle \equiv 2 p^{2} P_{1 / 2}$ and $\left|i^{\prime}\right\rangle \equiv 2 p^{2} P_{3 / 2}$ in Fig. 1.) Using the appropriate Clebsch-Gordon coefficients to evaluate the terms $\left\langle\downarrow, \uparrow\left|\vec{d} \cdot E_{j} \hat{\epsilon}_{j}\right| i\right\rangle$ in Eq. (2.3), we find the carrier Rabi frequency to be

$$
\Omega_{\downarrow, \uparrow}=\frac{g_{b} g_{r}}{\sqrt{6}}\left[b_{0} r_{+}+b_{-} r_{0}\right] \frac{\omega_{F}}{\Delta\left(\Delta-\omega_{F}\right)},
$$

where $\Delta$ is the detuning of the Raman beams from the ${ }^{2} P_{1 / 2}$ level $\left(\Delta_{i}\right.$ in the figure), $\omega_{F} / 2 \pi(\simeq 198 \mathrm{GHz})$ is the fine structure splitting, $\omega_{0} / 2 \pi \simeq 1.25 \mathrm{GHz}$, and $g_{b, r} \equiv E_{b, r}\left|\left\langle{ }^{2} P_{3 / 2}, F=3, m_{F}=3\left|\vec{d} \cdot \hat{\sigma}_{+}\right| \downarrow\right\rangle\right| / 2 \hbar$.

In some cases we will also find it useful to adjust the Raman beam difference frequency to a multiple of the mode oscillation frequency. When $\omega_{b}-\omega_{r} \simeq \omega_{z}$, the interaction (in the Lamb-Dicke limit) can be written

$$
H_{I}\left(m_{S}\right) \simeq \hbar \Omega\left(m_{S}\right) e^{-i\left(\omega_{b}-\omega_{r}\right) t}\left[1+i \eta\left(a e^{-i \omega_{z} t}+a^{\dagger} e^{i \omega_{z} t}\right)\right]+\text { h.c. }
$$


where

$$
\Omega\left(m_{S}\right) \equiv \frac{e^{i\left(\phi_{b}-\phi_{r}\right)}}{4 \hbar^{2}} \sum_{i}\left\langle m_{S}\left|\vec{d} \cdot E_{r} \hat{\epsilon}_{r}\right| i\right\rangle\left\langle i\left|\vec{d} \cdot E_{b} \hat{\epsilon}_{b}\right| m_{S}\right\rangle / \Delta_{i}
$$

with $m_{S} \in\{\downarrow, \uparrow\}$. Assuming $\omega_{b}-\omega_{r}=\omega_{z}$, and approximating Eq. (2.7) with the resonant term, an oscillating, spatially uniform force is created that can act as a displacement operator (Meekhof et al. 1996). It takes the form (Wineland et al. 1998)

$$
D(t)=D\left(\eta \Omega\left(m_{S}\right) t\right)=e^{\left(\eta \Omega\left(m_{S}\right) t\right) a^{\dagger}-\left(\eta \Omega\left(m_{S}\right) t\right)^{*} a} .
$$

When $\Omega(\downarrow) \neq \Omega(\uparrow)$, we can create logic gates as described in the example below.

\section{(c) Stark shifts}

In the above discussion we have neglected Stark shifts of the levels caused by the non-resonant laser beam electric fields. In the limit that $\Delta_{i} \gg \gamma$, the Stark shift from the $j$ th beam on the $m_{S}$ level is given by

$$
\delta\left(m_{S}, j\right)=\frac{1}{4 \hbar^{2}} \sum_{i}\left|\left\langle m_{S}\left|\vec{d} \cdot E_{j} \hat{\epsilon}_{j}\right| i\right\rangle\right|^{2} / \Delta_{i}
$$

In general, both levels $|\downarrow\rangle$ and $|\uparrow\rangle$ are shifted by both laser beams. For the $|2,2\rangle=$ $|\downarrow\rangle \leftrightarrow|1,1\rangle=|\uparrow\rangle^{9} \mathrm{Be}^{+}$Raman transition example, the net Stark shift is

$$
\begin{array}{r}
\delta(\uparrow)-\delta(\downarrow)=g_{b}^{2}\left[\frac{\left(\frac{1}{6} b_{-}^{2}+\frac{1}{3} b_{0}^{2}+\frac{1}{2} b_{+}^{2}\right)}{\Delta+\omega_{0}}+\frac{\left(\frac{5}{6} b_{-}^{2}+\frac{2}{3} b_{0}^{2}+\frac{1}{2} b_{+}^{2}\right)}{\Delta-\omega_{F}+\omega_{0}}\right. \\
\left.-\frac{\left(\frac{2}{3} b_{-}^{2}+\frac{1}{3} b_{0}^{2}\right)}{\Delta}-\frac{\left(\frac{1}{3} b_{-}^{2}+\frac{2}{3} b_{0}^{2}+b_{+}^{2}\right)}{\Delta-\omega_{F}}\right]+g_{r}^{2}\left[\frac{\left(\frac{1}{6} r_{-}^{2}+\frac{1}{3} r_{0}^{2}+\frac{1}{2} r_{+}^{2}\right)}{\Delta}\right. \\
\left.+\frac{\left(\frac{5}{6} r_{-}^{2}+\frac{2}{3} r_{0}^{2}+\frac{1}{2} r_{+}^{2}\right)}{\Delta-\omega_{F}}-\frac{\left(\frac{2}{3} r_{-}^{2}+\frac{1}{3} r_{0}^{2}\right)}{\Delta-\omega_{0}}-\frac{\left(\frac{1}{3} r_{-}^{2}+\frac{2}{3} r_{0}^{2}+r_{+}^{2}\right)}{\Delta-\omega_{0}-\omega_{F}}\right] .
\end{array}
$$

In Eq. (2.11) we have assumed that the (quantizing) magnetic field is small enough that Zeeman shifts can be neglected and that the ${ }^{2} P_{1 / 2}$ and ${ }^{2} P_{3 / 2}$ hyperfine splittings are negligible comparable to $\Delta_{i}$ (The hyperfine splitting of the ${ }^{2} P_{1 / 2}$ level is approximately $237 \mathrm{MHz}$ and that of the ${ }^{2} P_{3 / 2}$ level is less than $1 \mathrm{MHz}$.). In the limit that we can also assume $\omega_{0} \ll \Delta, \omega_{F}$, Eq. (2.11) reduces to

$$
\delta(\uparrow)-\delta(\downarrow) \simeq\left[g_{b}^{2}\left(b_{-}^{2}-b_{+}^{2}\right)+g_{r}^{2}\left(r_{-}^{2}-r_{+}^{2}\right)\right]\left[\frac{\omega_{F}}{2 \Delta\left(\Delta-\omega_{F}\right)}\right] .
$$

In this approximation, we see that the Stark shifts vanish if we use linearly polarized light for the Raman beams. Moreover, if we use the exact expression for $\delta(\uparrow)-\delta(\downarrow)$, we find that the Stark shift can be tuned to zero by adjusting $r_{-}-r_{+}$and/or $b_{-}-b_{+}$to small non-zero values. 


\section{(d) Spontaneous emission}

A fundamental limitation to the coherence of atomic qubits is spontaneous emission. When the qubits are formed from ground-state hyperfine levels, memory is not affected by spontaneous emission since these levels have very long radiative lifetimes. The problem arises from spontaneous emission during Raman transitions (see for example Plenio and Knight 1997, Di Fidio and Vogel 2000, Budini et al. 2002). As an estimate of the decoherence rate due to spontaneous emission we calculate the total spontaneous emission rate $R_{S E}$ from the $2 p$ levels. This rate is equal to the sum of the probabilities $P_{i}$ that each intermediate (excited) state is occupied times its decay rate $\gamma_{i}$ :

$$
R_{S E}=\sum_{i} \gamma_{i} P_{i}=\frac{1}{4 \hbar^{2}} \sum_{i} \sum_{\{j=r, b\}} \sum_{\left\{m_{S}=\downarrow, \uparrow\right\}} P_{m_{S}} \gamma_{i}\left|\left\langle m_{S}\left|\vec{d} \cdot E_{j} \hat{\epsilon}_{j}\right| i\right\rangle\right|^{2} / \Delta_{i}^{2}
$$

where $P_{m_{S}}$ is the probability of being in the $m_{S}$ ground state. For our ${ }^{9} \mathrm{Be}^{+}$example, we find

$$
\begin{gathered}
R_{S E}=\gamma P_{\downarrow}\left[\frac{g_{b}^{2}\left(\frac{2}{3} b_{-}^{2}+\frac{1}{3} b_{0}^{2}\right)}{\Delta^{2}}+\frac{g_{b}^{2}\left(\frac{1}{3} b_{-}^{2}+\frac{2}{3} b_{0}^{2}+b_{+}^{2}\right)}{\left(\Delta-\omega_{F}\right)^{2}}+\frac{g_{r}^{2}\left(\frac{2}{3} r_{-}^{2}+\frac{1}{3} r_{0}^{2}\right)}{\left(\Delta-\omega_{0}\right)^{2}}\right. \\
\left.+\frac{g_{r}^{2}\left(\frac{1}{3} r_{-}^{2}+\frac{2}{3} r_{0}^{2}+r_{+}^{2}\right)}{\left(\Delta-\omega_{0}-\omega_{F}\right)^{2}}\right]+\gamma P_{\uparrow}\left[\frac{g_{b}^{2}\left(\frac{1}{6} b_{-}^{2}+\frac{1}{3} b_{0}^{2}+\frac{1}{2} b_{+}^{2}\right)}{\left(\Delta+\omega_{0}\right)^{2}}\right. \\
\left.+\frac{g_{b}^{2}\left(\frac{5}{6} b_{-}^{2}+\frac{2}{3} b_{0}^{2}+\frac{1}{2} b_{+}^{2}\right)}{\left(\Delta-\omega_{F}+\omega_{0}\right)^{2}}+\frac{g_{r}^{2}\left(\frac{1}{6} r_{-}^{2}+\frac{1}{3} r_{0}^{2}+\frac{1}{2} r_{+}^{2}\right)}{\Delta^{2}}+\frac{g_{r}^{2}\left(\frac{5}{6} r_{-}^{2}+\frac{2}{3} r_{0}^{2}+\frac{1}{2} r_{+}^{2}\right)}{\left(\Delta-\omega_{F}\right)^{2}}\right],
\end{gathered}
$$

where $\gamma / 2 \pi=19.4 \mathrm{MHz}$. Of course, we want to minimize the probability of spontaneous emission during qubit operations. As one measure of this, we can calculate the probability of spontaneous emission during the time $\tau_{\pi}$ required to carry out a $\pi$ pulse on the carrier transition, given by $\Omega \tau_{\pi}=\pi / 2$ (Eqs. (2.4) and (2.5)). Since we also want to suppress Stark shifts, we take $b_{-} \simeq b_{+}$and $r_{-} \simeq r_{+}$. During the $\pi$ transition, $\left\langle P_{\downarrow}\right\rangle=\left\langle P_{\uparrow}\right\rangle=1 / 2$. With these assumptions, Eq. (2.14) gives the probability of spontaneous emission during a carrier $\pi$ pulse as

$$
P_{S E}=R_{S E} \tau_{\pi} \simeq \frac{\pi \gamma}{6\left|\Omega_{\downarrow, \uparrow}\right|}\left(g_{b}^{2}+g_{r}^{2}\right)\left[\frac{1}{\Delta^{2}}+\frac{2}{\left(\Delta-\omega_{F}\right)^{2}}\right] .
$$

This expression is minimized with the choices $\Delta=(\sqrt{2}-1) \omega_{F} \simeq 82 \mathrm{GHz}, g_{b}=g_{r}$, and $b_{0}=1, r_{+}=r_{-}=1 / \sqrt{2}\left(b_{+}=b_{-}=r_{0}=0\right)$ giving the value $P_{S E}=$ $(8 \pi / \sqrt{6})\left(\gamma / \omega_{F}\right) \simeq 0.001$. This probability will be increased by $1 / \eta$ for sideband transitions (in the Lamb-Dicke limit). For this reason, ${ }^{9} \mathrm{Be}^{+}$will not be a good choice for the ultimate qubit since probabilities for errors during a gate operation must be on the order of $10^{-4}$ or smaller to be able to incorporate error correction into long computations (Steane 2002).

To suppress the effects of spontaneous emission, we want an ion with a small ratio of spontaneous decay rate $\gamma$ to fine structure splitting (see for example, Wineland 2002). To make a straightforward comparison between different ions, we calculate the probability of spontaneous emission for various ions with half odd-integer nuclear spin $I$ for a $\pi$ pulse on the carrier of the $\left|F=I-1 / 2, m_{F}=0\right\rangle \leftrightarrow \mid F=$ 
Table 1. Probability of spontaneous emission $P_{S E}$ during a two-photon stimulated-Raman $\pi$ pulse on the carrier of the ${ }^{2} S_{1 / 2},\left|F=I-1 / 2, m_{F}=0\right\rangle \leftrightarrow\left|F=I+1 / 2, m_{F}=0\right\rangle$ transition for various ions of interest in quantum computing. ( $I$ is the nuclear spin, $\nu_{F} \equiv \omega_{F} / 2 \pi$, $\nu_{0} \equiv \omega_{0} / 2 \pi$.)

\begin{tabular}{ccccccc}
\hline ion & $I$ & $\gamma / 2 \pi(\mathrm{MHz})$ & $\nu_{F}(\mathrm{THz})$ & $\nu_{0}(\mathrm{GHz})$ & $\left|\delta_{0 \leftrightarrow 0} / \Omega_{0 \leftrightarrow 0}\right|$ & $P_{S E}$ \\
\hline${ }^{9} \mathrm{Be}^{+}$ & $3 / 2$ & 19.4 & 0.198 & 1.25 & $3.6 \times 10^{-2}$ & $8.7 \times 10^{-4}$ \\
${ }^{25} \mathrm{Mg}^{+}$ & $5 / 2$ & 43 & 2.75 & 1.79 & $3.6 \times 10^{-3}$ & $1.4 \times 10^{-4}$ \\
${ }^{43} \mathrm{Ca}^{+}$ & $7 / 2$ & 22.4 & 6.7 & 3.26 & $2.8 \times 10^{-3}$ & $3.0 \times 10^{-5}$ \\
${ }^{67} \mathrm{Zn}^{+}$ & $5 / 2$ & 76 & 26.2 & 7.2 & $1.6 \times 10^{-3}$ & $2.6 \times 10^{-5}$ \\
${ }^{87} \mathrm{Sr}^{+}$ & $9 / 2$ & 21.7 & 24 & 5.00 & $1.2 \times 10^{-3}$ & $8.0 \times 10^{-6}$ \\
${ }^{113} \mathrm{Cd}^{+}$ & $1 / 2$ & 44.2 & 74 & 15.2 & $1.2 \times 10^{-3}$ & $5.3 \times 10^{-6}$ \\
${ }^{199} \mathrm{Hg}^{+}$ & $1 / 2$ & 54.7 & 274 & 40.5 & $8.4 \times 10^{-4}$ & $1.8 \times 10^{-6}$ \\
\hline
\end{tabular}

$\left.I+1 / 2, m_{F}=0\right\rangle$ transition. The Rabi rate for these transitions will be independent of $I$. Moreover, the frequencies of these transitions deviate from their values at magnetic field $\vec{B}=0$ by an amount proportional to $|\vec{B}|^{2}$ and are therefore first-order independent of field fluctuations as $|\vec{B}| \rightarrow 0$. Again making the approximations $\omega_{0}, \gamma \ll \omega_{F},|\Delta|$, we find

$$
\Omega_{0 \leftrightarrow 0}=\frac{g_{b} g_{r} \omega_{F}}{3 \Delta\left(\Delta-\omega_{F}\right)}\left(b_{-} r_{-}-b_{+} r_{+}\right)
$$

and

$$
R_{S E}=\gamma \frac{g_{b}^{2}+g_{r}^{2}}{3}\left[\frac{1}{\Delta^{2}}+\frac{2}{\left(\Delta-\omega_{F}\right)^{2}}\right] .
$$

For simplicity we have taken the value of $\gamma$ to be that corresponding to the relevant ${ }^{2} P_{1 / 2}$ level, we have assumed pure ${ }^{2} P_{1 / 2}$ and ${ }^{2} P_{3 / 2}$ configurations, and we have included coupling to only this pair of fine-structure levels. These approximations should be most accurate for the lighter ions. We can minimize $R_{S E} /\left|\Omega_{0 \leftrightarrow 0}\right|$ by choosing $\Delta=(\sqrt{2}-1) \omega_{F}, g_{b}=g_{r}$, and the experimentally convenient choice of orthogonal linear polarizations for the Raman beams $\left(b_{-}=r_{-}=b_{+}=-r_{+}=\right.$ $1 / \sqrt{2}$ ). This leads to a probability of spontaneous emission during a carrier $\pi$ pulse given by $P_{S E}=2 \sqrt{2} \pi \gamma / \omega_{F}$. The Stark shift of the transition is equal to

$$
\delta_{0 \leftrightarrow 0}=-\frac{\left(g_{b}^{2}+g_{r}^{2}\right) \omega_{0}}{3}\left[\frac{1}{\Delta^{2}}+\frac{2}{\left(\Delta-\omega_{F}\right)^{2}}\right],
$$

which is independent of polarization. For the conditions above, we find $\delta_{0 \leftrightarrow 0}=$ $-4 \sqrt{2}\left|\Omega_{0 \leftrightarrow 0}\right| \omega_{0} / \omega_{F}$. In Table 1 we tabulate $\left|\delta_{0 \leftrightarrow 0} / \Omega_{0 \leftrightarrow 0}\right|$ and $P_{S E}$ for a few ions of interest.

In the Lamb-Dicke limit, sideband transitions and two-qubit gates will necessarily have a higher probability of spontaneous emission since the Rabi frequencies for these processes scale as the carrier frequency times $\eta$, whereas the spontaneous emission rate will remain unchanged. Therefore, from Table 1 we see that, in order to suppress the probability of spontaneous emission during Raman transitions, a heavy ion such as $\mathrm{Cd}^{+}$(Blinov et al. 2002) will ultimately be required. Moreover, even for heavy ions, there is not much headroom on fidelity to reach fault tolerance. Therefore, for fault-tolerant quantum computation, values of $\eta$ should not be too 
small compared to unity; that is, the Lamb-Dicke limit will not be a good approximation. This implies the need for precise control of the motion (e.g., ground state cooling) since the gate Rabi rates will depend on the motional states. In addition, schemes that incorporate error resistant methods such as adiabatic passage, spin echo, and composite pulses like those used in NMR (Cummings et al. 2002, Jones 2002) will have to be used sparingly, if at all, since these techniques increase the probability of spontaneous emission for each logical operation.

In principle, we can relax these restrictions by making $|\Delta| \gg \omega_{F S}$. In the above discussion we have estimated decoherence using the total rate of spontaneous emission (Eqs. (2.14) and (2.17)). However, when $|\Delta| \gg \omega_{F S}$, spontaneous emission is dominated by Raleigh scattering and the decoherence rate will be suppressed compared to the total spontaneous emission rate (see, for example, Cline et al. (1994)). The cost for making $|\Delta| \gg \omega_{F S}$ is that the Raman laser beam intensities must increase as $\Delta^{2}$ to maintain the same Rabi frequency. Moreover, for large values of $\omega_{F S}$, we must consider the decohering effects of spontaneous Raman scattering from levels other than the two fine-structure levels considered here.

Finally, we note that the spontaneous emission problem could be essentially eliminated by driving single-photon hyperfine carrier and sideband transitions at frequencies $\omega_{0}$ and $\omega_{0} \pm \omega_{z}$. However, it appears that in practice, currently attainable field gradients (necessary for sideband transitions) would lead to relatively small values of $\eta$ and therefore slow sideband transition Rabi rates (see, for example Wineland et al. 1992 and Mintert and Wunderlich 2001).

\section{(e) State initialization and detection}

To initialize the qubits for each experiment, we use a combination of internalstate optical pumping to pump to the $|\downarrow\rangle$ state and laser cooling to optically pump the motional modes to their ground states (Monroe et al. 1995a, King et al. 1998, Roos et al. 1999). As in many atomic physics experiments, the observable in the ion trap experiments is the ion's spin qubit state. We can efficiently distinguish $|\downarrow\rangle$ from $|\uparrow\rangle$ using a cycling transition to implement "quantum jump" detection (Blatt \& Zoller 1988).

\section{Gates}

The previous sections summarize the basic sources of entanglement in ion experiments from which universal logic gates have been constructed. For example, a CNOT and $\pi$-phase gate between the motion and spin qubit for a single ion has been realized by Monroe et al. (1995b), Wineland et al. (1998), and DeMarco et al. (2002). Also, using the scheme suggested by Sørensen and Mølmer (1999, 2000) and Solano et al. (1999), the NIST group realized a universal gate between two spin qubits (Sackett et al. 2000, Kielpinski et al. 2001). Compared to the original Cirac and Zoller gate (1995), this last gate has the practical advantages that (1) laserbeam focusing (for individual ion addressing) is not required, (2) it can be carried out in one step, (3) it does not require use of an additional internal state, and (4) it does not require precise control of the motional state (as long as the Lamb-Dicke

limit is satisfied). From this gate, a CNOT gate can be constructed (Sørensen and 
Mølmer (1999)). Below, we discuss a $(\pi)$ phase gate based on a spin-dependent displacement operator.

\section{(a) Geometrical phase gate}

We have recently realized a $(\pi)$ phase gate between two spin qubits (Leibfried et al. 2002) that carried out the transformations: $|\downarrow\rangle|\downarrow\rangle \rightarrow|\downarrow\rangle|\downarrow\rangle,|\downarrow\rangle|\uparrow\rangle \rightarrow|\downarrow\rangle|\uparrow\rangle$, $|\uparrow\rangle|\downarrow\rangle \rightarrow|\uparrow\rangle|\downarrow\rangle$, and $|\uparrow\rangle|\uparrow\rangle \rightarrow-|\uparrow\rangle|\uparrow\rangle$. Consider constructing a closed path in phase space (for a particular motional mode) so that the state returns to its original position. We can derive the effects of this transformation by constructing the closed path from a series of successive applications of the displacement operator (see for example, Walls \& Milburn 1994) with infinitesimal displacements. The net effect is that the wave function describing the ion (or ions) acquires an overall phase shift that depends on the area enclosed by the path.

The second element required for the gate is to make the area of the path be spin-dependent. This is accomplished by making the displacement in phase space with a spin-dependent optical dipole force. This, at first, seems to be impossible if we require that the net Stark shift between the $|\downarrow\rangle$ and $|\uparrow\rangle$ states be equal to zero since optical dipole forces and Stark shifts are proportional. However, we can make the time-averaged Stark shift $\langle\delta(\uparrow)-\delta(\downarrow)\rangle$ be equal to zero for durations much greater than $2 \pi / \omega_{z}$ and still realize a spin dependent displacement operator if we choose the polarizations of the Raman laser beams appropriately. The basic idea is that we make the dipole force (sinusoidally varying at frequency $\omega_{z}$ ) have a different phase for the $|\downarrow\rangle$ and $|\uparrow\rangle$ states.

For simplicity, assume both Raman beams are linearly polarized with polarization perpendicular to the quantizing magnetic field. Also assume that the redRaman beam polarization has an angle $\kappa$ with respect to that of the blue-Raman beam. Hence we have $E_{b} \hat{\epsilon_{b}}=E_{b}\left(b_{-} \hat{\sigma}_{-}+b_{+} \hat{\sigma}_{+}\right)$and $E_{r} \hat{\epsilon_{r}}=E_{r}\left(r_{-} \hat{\sigma}_{-}+r_{+} \hat{\sigma}_{+} e^{i 2 \kappa}\right)$ where, as before, we take $b_{-} \simeq b_{+} \equiv b$ and $r_{-} \simeq r_{+} \equiv r$ to make $\langle\delta(\uparrow)-\delta(\downarrow)\rangle=0$. For our ${ }^{9} \mathrm{Be}^{+}$example, the interaction Hamiltonian is given by Eqs. (2.7) and (2.8), with

$\Omega(\downarrow)=g_{r} g_{b} b r\left[\frac{2 / 3}{\Delta}+\frac{1 / 3+e^{i 2 \kappa}}{\Delta-\omega_{F}}\right], \quad \Omega(\uparrow)=g_{r} g_{b} b r\left[\frac{1 / 6+1 / 2 e^{i 2 \kappa}}{\Delta}+\frac{5 / 6+1 / 2 e^{2 i \kappa}}{\Delta-\omega_{F}}\right]$.

By choosing the polarizations orthogonal $(\kappa=\pi / 2)$ and $\Delta=(\sqrt{2}-1) \omega_{F}$, we find

$$
\Omega(\downarrow)=\frac{2 g_{b} g_{r} b r}{3(3 \sqrt{2}-4) \omega_{F}}=-2 \Omega(\uparrow) .
$$

Hence, the dipole force on the $|\downarrow\rangle$ state is twice that on the $|\uparrow\rangle$ state and in the opposite direction. (This same displacement operator has been used previously to create Schrödinger cat states of a single ion (Myatt et al. 2000).) To implement this gate on two ions, the Raman transition beams were separated in frequency by $\sqrt{3} \omega_{z}+\delta$, where $\sqrt{3} \omega_{z}$ is the stretch mode frequency for two ions aligned along the $z$ axis and $\delta$ is a small detuning $\left(\ll \omega_{z}\right)$. The separation of the ions was adjusted to be an integer multiple of $2 \pi / \Delta k$ so that the optical-dipole force on each ion was in the same direction if the ions were in the same spin state but in opposite directions if the spin states was different (Eq. (3.2)). This had the effect that the 


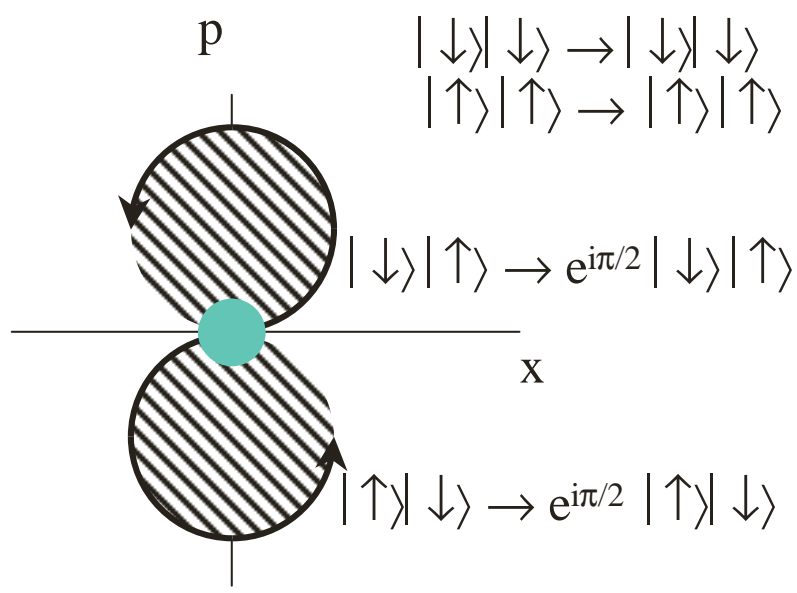

Figure 2. Schematic representation of the displacements of the axial stretch-mode amplitude in phase space for the four basis states of the two spin qubits. The detuning and amplitude of the displacements are chosen to give a $\pi / 2$ phase shift on the $|\downarrow\rangle|\uparrow\rangle$ and $|\uparrow\rangle|\downarrow\rangle$ states while the $|\downarrow\rangle|\downarrow\rangle$ and $|\uparrow\rangle|\uparrow\rangle$ states are unaffected because the optical dipole forces for these states do not couple to the stretch mode.

application of the laser beams to the $|\downarrow\rangle|\uparrow\rangle$ and $|\uparrow\rangle|\downarrow\rangle$ states caused excitation on the stretch mode but the motion was not excited when the ions were in the $|\downarrow\rangle|\downarrow\rangle$ or $|\uparrow\rangle|\uparrow\rangle$ states. The detuning $\delta$ and duration of the displacement pulses $(2 \pi / \delta)$ were chosen to make one complete (circular) path in phase space with an area that gave a phase shift of $\pi / 2$ on the $|\downarrow\rangle|\uparrow\rangle$ and $|\uparrow\rangle|\downarrow\rangle$ states (Fig. 2). Under these conditions, the overall transformation was: $|\downarrow\rangle|\downarrow\rangle \rightarrow|\downarrow\rangle|\downarrow\rangle,|\downarrow\rangle|\uparrow\rangle \rightarrow e^{i \pi / 2}|\downarrow\rangle|\uparrow\rangle$, $|\uparrow\rangle|\downarrow\rangle \rightarrow e^{i \pi / 2}|\uparrow\rangle|\downarrow\rangle$, and $|\uparrow\rangle|\uparrow\rangle \rightarrow|\uparrow\rangle|\uparrow\rangle=e^{i \pi} e^{-i \pi}|\uparrow\rangle|\uparrow\rangle$. Therefore, this operator acts like the product of an operator that applies a $\pi / 2$ phase shift to the $|\uparrow\rangle$ state on each ion separately (a non-entangling gate) and $\pi$ phase gate between the two ions. The $\pi / 2$ phase shifts can be removed by applying additional single qubit rotations or accounted for in software. (In an algorithm carried out by a series of singlequbit rotations and two-qubit phase gates, the extra phase shifts can be removed by appropriately shifting the phase of subsequent or prior single-qubit rotations.)

This phase gate is a particular case of the more general formalism developed by Milburn et al. (1999), Sørensen and Mølmer (1999), and Wang et al. (2001). Compared to the original Cirac and Zoller gate (1995) this gate shares the same advantages as the Sørensen and Mølmer gate (2000) as well as some additional technical advantages (Leibfried et al. 2002).

\section{Whither quantum computation?}

Although simple operations among a few ion qubits have been demonstrated, a viable quantum computer must look towards scaling to very large numbers of qubits. As the number of ions in a trap increases, several difficulties are encountered. For example, the addition of each ion adds three vibrational modes. It soon becomes nearly impossible to spectrally isolate the desired vibrational mode unless the speed of operations is slowed to undesirable levels (see for example Wineland et al. 1998, Steane and Lucas, 2000). Furthermore, since error correction will most likely be 
incorporated into any large processor, it will be desirable to measure and reset ancilla qubits without disturbing the coherence of logical qubits. Since ion qubits are typically measured by means of state-dependent laser scattering, the scattered light from ancilla qubits held in a common trap may disturb the coherence of the logical qubits.

For these and other reasons, it appears that a scalable ion-trap system must incorporate arrays of interconnected traps, each holding a small number of ions. The information carriers between traps might be photons (Cirac et al. 1997, Pellizzari 1997, De Voe 1998), or ions that are moved between traps in the array. In the latter case, a "head" ion held in a movable trap could carry the information by moving from site-to-site as in the proposal of Cirac and Zoller (2000). Similarly, as has been proposed at NIST, we could shuttle ions around in an array of interconnected traps (Wineland et al. 1998, Kielpinski et al. 2002). In this last scheme, the idea is to move ions between nodes in the array by applying time-dependent potentials to "control" electrode segments. To perform logic operations between selected ions, these ions are transferred into an "accumulator" trap for the gate operation. Before the gate operation is performed, it may be necessary to sympathetically re-cool the qubit ions with another ion species. Subsequently, these ions are moved to memory locations or other accumulators. This strategy always maintains a relatively small number of motional modes that must be considered and minimizes the problems of ion/laser-beam addressing using focused laser beams. Such arrays also enable highly parallel processing and ancilla qubit readout in separate trapping regions so that the logical ions are shielded from the scattered laser light. Some of the initial steps towards this scheme have been reported by Rowe et al. (2002).

The obstacles to building a large-scale quantum computer appear to be technical rather than fundamental. However since it will be a long time before a useful processor is constructed, it is helpful to have some intermediate goals upon which projections about scaling can be made. Examples goals might be the demonstration of repetitive error correction (see for example, Nielsen and Chuang 2000) and the realization of gates at the fault-tolerant level of fidelity (Steane 2002).

\section{Acknowledgements}

We thank Marie Jensen and Windell Oskay for helpful comments on the manuscript. This work was supported by the U. S. National Security Agency (NSA) and Advanced Research and Development Activity (ARDA) under Contract No. MOD7171.00, and the U. S. Office of Naval Research (ONR). The article is a contribution of NIST and not subject to U.S. copyright.

\section{References}

Barenco, A., Bennett, C. H., Cleve, R., DiVincenzo, D. P., Margolus, N., Shor, P., Sleator, T., Smolin, J., Weinfurter, H. 1995 Elementary gates for quantum computation. Phys. Rev. A 52, 3457-3467.

Blatt, R., Zoller, P. 1988 Quantum jumps. Eur. J. Phys. 9, 250-279.

Blinov, B. B., Deslauriers, L., Lee, P., Madsen, M. J., Miller, R., Monroe, C. 2002 Sympathetic cooling of trapped $\mathrm{Cd}^{+}$isotopes. Phys. Rev. A 65, 040304-1-4. 
Budini, A. A., de Matos Filho, R. L., Zagury, N. 2002 Localization and dispersive-like decoherence in vibronic states of a trapped ion. Phys. Rev. A 65, 041402-1-4.

Cirac, J. I., Zoller, P. 1995 Quantum computation with cold, trapped ions. Phys. Rev. Lett. 74, 4091-4094.

Cirac, J. I., Zoller, P. 2000 A scalable quantum computer with ions in an array of microtraps. Nature 404, 579-581.

Cirac, J. I., Zoller, P., Kimble, H. J., Mabuchi, H. 1997 Quantum state transfer and entanglement distribution among distant nodes in a quantum network. Phys. Rev. Lett. 78, 3221-3224.

Cline, R. A., Miller, J. D., Matthews, M. R., Heinzen, D. J. 1994 Spin relaxation of optically trapped atoms by light scattering. Opt. Lett. 19, 207-209.

Cummins, H. K., Llewellyn, G., Jones, J. A. 2002 Tackling systematic errors in quantum logic gates with composite rotations. quant-ph/0208092.

DeMarco, B., Ben-Kish, A., Leibfried, D., Meyer, V., Rowe, M., Jelenković, B. M., Itano, W. M., Britton, J., Langer, C., Rosenband, T., Wineland, D. J. Experimental demonstration of a controlled-not wave-packet gate. quant-ph/0208180, Phys. Rev. Lett., to be published.

DeVoe, R. G. 1998 Elliptical ion traps and trap arrays for quantum computation. Phys. Rev. A 58, 910-914.

Di Fidio, C., Vogel, W. 2000 Damped Rabi oscillations of a cold trapped ion. Phys. Rev. A 62, 031802-1-4.

DiVincenzo, D. P. 1995 Two-bit gates are universal for quantum computation. Phys. Rev. A 51, 1015-1022.

DiVincenzo, D. P. 2001 The physical implementation of quantum computation. In S. L. Braunstein, H. K. Lo, eds., Scalable Quantum Computers. Wiley-VCH, Berlin, 1-13.

Jaksch, D., Cirac, J. I., Zoller, P., Rolston, S. L., Côté, R., Lukin, M. D. 2000 Fast quantum gates for neutral atoms. Phys. Rev. Lett. 85, 2208-2211.

Jones, J. A. 2002 Robust Ising gates for practical quantum computation. quantph/0209049.

Kielpinski, D., Meyer, V., Rowe, M. A., Sackett, C. A., Itano, W. M., Monroe, C., Wineland, D. J. 2001 A decoherence-free quantum memory using trapped ions. Science 291, 1013-1015.

Kielpinski, D., Monroe, C., Wineland, D. J. 2002 Architecture for a large-scale ion-trap quantum computer. Nature 417, 709-711.

King, B. E., Wood, C. S., Myatt, C. J., Turchette, Q. A., Leibfried, D., Itano, W. M., Monroe, C., Wineland, D. J. 1998 Cooling the collective motion of trapped ions to initialize a quantum register. Phys. Rev. Lett. 81, 1525-1528.

Leibfried, D., DeMarco, B., Meyer, V., Lucas, D., Barrett, M., Britton, J., Itano, W. M., Jelenković, B., Langer, C., Rosenband, T., Wineland, D. J. 2002 Experimental demonstration of a robust, high-fidelity geometrical two ion-qubit phase gate. submitted for publication.

Meekhof, D. M., Monroe, C., Itano, W. M., King, B. E., Wineland, D. J. 1996 Generation of nonclassical motional states of a trapped atom. Phys. Rev. Lett. 76, 1796-1799.

Milburn, G. J., Schneider, S., James, D. F. 2000 Ion trap quantum computing with warm ions. In S. L. Braunstein, H. K. Lo, P. Kok, eds., Scalable Quantum Computers. WileyVCH, Berlin, 31-40.

Mintert, F., Wunderlich, C. 2001 Ion-trap quantum logic using long-wavelength radiation. Phys. Rev. Lett. 87, 257904-1-4.

Monroe, C., Meekhof, D. M., King, B. E., Jefferts, S. R., Itano, W. M., Wineland, D. J., Gould, P. 1995a Resolved-sideband Raman cooling of a bound atom to the 3d zero-point energy. Phys. Rev. Lett. $\mathbf{7 5}$.

Article submitted to Royal Society 
Monroe, C., Meekhof, D. M., King, B. E., Itano, W. M., Wineland, D. J. 1995b Demonstration of a fundamental quantum logic gate. Phys. Rev. Lett. 75, 4714-4717.

Monroe, C., Leibfried, D., King, B. E., Meekhof, D. M., Itano, W. M., Wineland, D. J. 1997 Simplified quantum logic with trapped ions. Phys. Rev. A 55, R2489-2491.

Myatt, C. J., King, B. E., Turchette, Q. A., Sackett, C. A., Kielpinski, D., Itano, W. M., Monroe, C., Wineland, D. J. 2000 Decoherence of quantum superpositions through coupling to engineered reservoirs. Nature 403, 269-273.

Nielsen, M. A., Chuang, I. L. 2000 Quantum Computation and Quantum Information. Cambridge Univ. Press, Cambridge, 1st ed.

Pellizzari, T. 1997 Phys. Rev. Lett. 79, 5242-5245.

Plenio, M. B., Knight, P. L. 1997 Decoherence limits to quantum computation using trapped ions. Proc. R. Soc. Lond. A 453, 2017-2041.

Raimond, J. M., Brune, M., Haroche, S. 2001 Manipulating quantum entanglement with atoms and photons in a cavity. Rev. Mod. Phys. 73, 565-582.

Roos, C., Zeiger, T., Rohde, H., Nägerl, H. C., Eschner, J., Leibfried, D., Schmidt-Kaler, F., Blatt, R. 1999 Quantum state engineering on an optical transition and decoherence in a Paul trap. Phys. Rev. Lett. 83, 4713-4717.

Rowe, M. A., Ben-Kish, A., DeMarco, B., Leibfried, D., Meyer, V., Beall, J., Britton, J., Hughes, J., Itano, W. M., Jelenković, B., Langer, C., Rosenband, T., Wineland, D. J. 2002 Transport of quantum states and separation of ions in a dual RF ion trap. Quant. Inform. Comp. 2, 257-271.

Sackett, C. A., Kielpinski, D., King, B. E., Langer, C., Meyer, V., Myatt, C. J., Rowe, M., Turchette, Q. A., Itano, W. M., Wineland, D. J., Monroe, C. 2000 Experimental entanglement of four particles. Nature 404, 256-258.

Solano, E., de Matos Filho, R. L., Zagury, N. 1999 Deterministic Bell states and measurement of the motional state of two trapped ions. Phys. Rev. A 59, 2539-2542.

Sørensen, A., Mølmer, K. 1999 Quantum computation with ions in thermal motion. Phys. Rev. Lett. 82, 1971-1974.

Sørensen, A., Mølmer, K. 2000 Entanglement and quantum computation with ions in thermal motion. Phys. Rev. A 62, 02231-1-11.

Steane, A. M. 2002 Overhead and noise threshold of fault-tolerant quantum error correction. quant-ph/0207119.

Steane, A. M., Lucas, D. M. 2000 Quantum computing with trapped ions, atoms and light. In S. L. Braunstein, H. K. Lo, P. Kok, eds., Scalable Quantum Computers. Wiley-VCH, Berlin, 69-88.

Walls, D. F., Milburn, G. J. 1994 Quantum Optics. Springer, Berlin, 1st ed.

Wang, X., Sørensen, A., Mølmer, K. 2001 Mulitbit gates for quantum computing. Phys. Rev. Lett. 86, 3907-3910.

Wineland, D. J. 2002 Trapped ions and quantum information processing. Proc. Fermi School on Quantum Computation, Nuovo Cimento, to be published .

Wineland, D. J., Bollinger, J. J., Itano, W. M., Moore, F. L., Heinzen, D. J. 1992 Spin squeezing and reduced quantum noise in spectroscopy. Phys. Rev. A 46, R6797-R6800.

Wineland, D. J., Monroe, C., Itano, W. M., Leibfried, D., King, B. E., Meekhof, D. M. 1998 Experimental issues in coherent quantum-state manipulation of trapped atomic ions. J. Res. Nat. Inst. Stand. Tech. 103, 259-328. 